\title{
Major signaling pathways and key mediators of macrophages in acute kidney injury (Review)
}

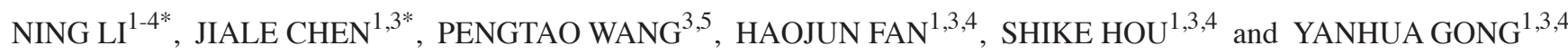 \\ ${ }^{1}$ Institute of Disaster Medicine, Tianjin University, Tianjin 300072; ${ }^{2}$ State Key Laboratory of Medicinal Chemical Biology, \\ Nankai University, Tianjin 300350; ${ }^{3}$ Tianjin Key Laboratory of Disaster Medicine Technology, Tianjin 300072; \\ ${ }^{4}$ Wenzhou Safety (Emergency) Institute, Tianjin University, Wenzhou, Zhejiang 325000; \\ ${ }^{5}$ Emergency Department, General Hospital of Tianjin Medical University, Tianjin 300070, P.R. China
}

Received November 26, 2020; Accepted March 17, 2021

DOI: $10.3892 / \mathrm{mmr} .2021 .12094$

\begin{abstract}
Acute kidney injury (AKI) has become a global public health problem with high morbidity and mortality rates, as well as high healthcare costs. Immune cells, particularly macrophages, which regulate tissue development, destroy pathogens, control homeostasis and repair wounds, play crucial and complex roles in AKI. In various types of AKI, numerous rapidly recruited monocytes and tissue-resident macrophages act in a coordinated manner. Thus, elucidating
\end{abstract}

Correspondence to: Professor Shike Hou or Professor Yanhua Gong, Institute of Disaster Medicine, Tianjin University, 92 Weijin Road, Nankai, Tianjin 300072, P.R. China

E-mail: houshike@tju.edu.cn

E-mail: gongyanhua@tju.edu.cn

*Contributed equally

Abbreviations: AKI, acute kidney injury; APN, acute pyelonephritis; AQP1, aquaporin 1; CCL5, C-C motif chemokine 5; CCR2, chemokine receptor 2; CCR5, chemokine receptor 5; CI-AKI, contrast-induced AKI; CKD, chronic kidney disease; CLP, cecal ligation and puncture; CXCL14, C-X-C motif chemokine ligand 14; DAMP, damage-associated molecular pattern; EPO, erythropoietin; EV, extracellular vesicles; ET, extracellular trap; Gpnmb, glycoprotein non-metastatic melanoma protein $b ; \mathrm{I} / \mathrm{R}$, ischemia/reperfusion; I/R-AKI, I/R-induced AKI; IL, interleukin; JAK, janus kinase; KIM-1, kidney injury molecule-1; Lcn-2, lipocalin-2; LPS, lipopolysaccharide; MAPK, mitogen-activated protein kinase; METs, macrophage extracellular traps; MIF, macrophage migration inhibitory factor; Mincle, macrophage-inducible C-type lectin; miR, microRNA; NETs, neutrophil extracellular traps; NF- $\kappa \mathrm{B}$, nuclear factor- $\kappa \mathrm{B}$; NGAL, neutropil gelatinase-associated lipocalin; PI3K, phosphatidylinositol-3 kinase; PTBA, 4-(phenylthio) butanoic acid; RA, retinoic acid; RI-AKI, rhabdomyolysis-induced AKI; RTECs, renal tubular epithelial cells; sCD163, soluble CD163; STAT, signal transducers and activators of transcription; TLR4, toll-like receptor 4; Trib1, tribbles homolog 1; TRX, thioredoxin-1

Key words: acute kidney injury, signal pathway, macrophagesensing mediators, macrophage-derived mediators the phenotypic and functional characteristics of macrophages in AKI is essential for identifying potential therapeutic targets. Macrophage-sensing mediators and macrophage-derived mediators participate in the major macrophage-related signaling pathways in AKI, which regulate macrophage polarization and determine disease progression. In conclusion, macrophages change their roles and regulatory mechanisms during the occurrence and development of AKI. The aim of the present review was to contribute to an improved understanding of AKI and to the identification of novel therapeutic targets for this condition.

\section{Contents}

1. Introduction

2. Signal transduction of macrophages in AKI

3. Macrophage-derived mediators in AKI

4. Macrophage-sensing mediators in AKI

5. Conclusions

\section{Introduction}

Trauma involves the regulation of immune homeostasis, the production and release of various damage-associated molecular patterns (DAMPs) and the activation of the innate immune system (1). The post-traumatic biological response is a complex physiological phenomenon involving multiple inflammatory and thrombotic mediators, including cytokines, chemokines, complement receptors, oxygen free radicals, inflammatory cells (neutrophils, monocytes and macrophages) and endothelial cells (2). Macrophages exist in mammalian tissues and have essential functions (3). Although its origin continues to be controversial, the embryonic origin of the vital tissue that resides in macrophages is now understood (4). Macrophages residing in the majority of tissues are long-lived cells derived from transient hematopoietic waves of erythro-myeloid progenitors that emerge in the yolk sac (5). Although macrophages were previously known for their properties of host defense and scavenging of apoptotic cells, it is increasingly recognized that macrophages play a variety of roles in tissue development, 
homeostasis control and wound repair $(6,7)$. Inflammatory monocytes recruited by different mechanisms and activated tissue-resident macrophages are critical regulatory cells of tissue repair, regeneration and fibrosis (8). Several of these functional features are essential for tissue injury and repair. These cells can not only aggravate tissue damage by generating reactive oxygen species and other toxic components, but also produce various growth factors, including VEGF- $\alpha$ and TGF- $\beta$, to promote cell proliferation and repair (9). In the early 1990s, a type of selectively activated macrophage (type M2) was identified, which was different from the classical activated inflammatory macrophage (type M1) (10). M2 macrophages are usually described by their anti-inflammatory and wound healing effects (11). Macrophages can affect the metabolic tissue and alter the metabolic pathways. This phenotypic transformation occurs in glucose and lipid metabolism (12). Macrophages are the principal participants of the immune response after tissue injury, and their phenotypes vary from pro-inflammatory (M1) to anti-inflammatory (M2) macrophages, which can promote wound healing and scar repair (13). In addition, macrophage polarization is a highly dynamic process that is easily modulated by its microenvironment (14). Furthermore, the reversibility of macrophage polarization also has an important therapeutic value, particularly in diseases caused by an M1/M2 imbalance (15).

Acute kidney injury (AKI) has become a global public health problem with high morbidity, and mortality rates, as well as high healthcare costs (16). AKI is a disease characterized by acutely decreased renal function, the etiology of which can be multifactorial and is associated with complex pathophysiological mechanisms (17). AKI has been associated with mortality after traumatic war injuries (18). The pathogenesis of AKI varies with different types of damage. In ischemia/reperfusion (I/R)-induced AKI (I/R-AKI), the loss of the brush border of renal tubular epithelial cells (RTECs) and cell polarization leads to tubular obstruction, cell necrosis and apoptosis (19). In cisplatin-induced AKI, cisplatin causes DNA damage and mitochondrial damage, leading to inflammation and cell death (20). In contrast-induced AKI (CI-AKI), the formation of vacuoles, swelling of cells and oxidative stress lead to acute necrosis (21). In sepsis-induced AKI, sepsis may lead to renal vasodilation caused by inducible nitric oxide synthase release (19). Apart from kidney dialysis, there is no treatment for AKI that can reliably improve survival, reduce injury or accelerate recovery (22). AKI participates in the regulation of immune system homeostasis, and numerous factors are involved, including erythropoietin (EPO), glycoprotein non-metastatic melanoma protein $B(\mathrm{Gpnmb})$, retinoic acid (RA), colony stimulating factor-1, myoglobin, dendritic cells, neutrophils and macrophages (23-25). A previous study demonstrated that macrophages are the principal effectors in AKI inflammatory responses (26). The dynamic roles and functional properties of macrophages in AKI are important for the identification of effective therapeutic targets (27). The activation and functional state of macrophages after renal injury are complex and diverse. Macrophages damage or repair renal tubules, and their role in interstitial fibrosis after renal injury varies with time and is determined by the type of renal injury (28). Due to the functional plasticity of macrophages (i.e., their ability to transform between the pro-inflammatory M1 and anti-inflammatory M2 phenotypes), macrophages play a complex role in the occurrence and development of AKI (29). Importantly, the polarization of macrophages may lead to the development of novel treatments to promote AKI repair (29). At present, it is considered that M2 macrophages and regulatory T cells are critical cells controlling inflammation, as well as tissue remodeling and repair following AKI (30). Previous studies have shown that M2 macrophage therapy can effectively reduce renal injury in AKI mice $(31,32)$. The increase in M2 macrophages may play a pivotal role in the initial damage of human AKI and in the transition from AKI to chronic kidney disease (CKD) (33). Notably, animal experiments have shown that total or partial macrophage depletion in AKI may be beneficial to kidney damage (34). For example, M1 depletion has been demonstrated to have a universal protective effect on AKI; however, the results caused by M2 depletion are controversial (34). The latest research shows that nattokinase and hydrogen-rich solution produce a therapeutic effect on the inflammatory response of AKI by regulating the activity of macrophages $(35,36)$. In summary, at present, the multifaceted role of macrophages in the occurrence and development of AKI remains open to further investigation.

\section{Signal transduction of macrophages in AKI}

Phosphatidylinositol-3 kinase (PI3K) signaling pathway. The PI3K pathway is one of the main signaling pathways that regulates macrophages, and it controls the critical switch between immune activation and inhibition in the process of inflammation (37). In addition, its contribution to macrophage polarization has gradually attracted the attention of numerous researchers, and been demonstrated to mediate the transformation of M2 macrophages $(38,39)$. Certain studies have revealed that PI $3 \mathrm{~K} \gamma$ plays a pivotal role in the polarization of macrophages and in the development of renal disease. Specifically, a lack of PI3K $\gamma$ leads to the polarization of M1 macrophages, resulting in an inflammatory environment $(40,41)$. A previous study raised concerns regarding the inhibition of PI3K/AKT/mTOR in the treatment of AKI (41). Furthermore, previous studies found that aquaporin 1 (AQP1) can prevent renal tissue damage in AKI induced by bacterial lipopolysaccharide (LPS) by mediating the immune response. AQP1 alleviates sepsis-induced AKI by successfully activating PI3K, eventually leading to macrophages polarization to the M2 phenotype (Fig. 1) (42). Therefore, targeting PI3K-dependent M2 macrophage polarization may constitute a novel therapeutic approach to reducing sepsis-induced AKI.

Nuclear factor $-\kappa B(N F-\kappa B)$ signaling pathway. $N F-\kappa \mathrm{B}$ is a key regulator of inherent and adjustable immunity, which is closely linked to the pathogenesis of AKI (43). Previous studies have shown that pharmacological inhibitors (CLI-095 and pyrrolidine dithiocarbamate) of toll-like receptor 4 (TLR4)/NF- $\kappa \mathrm{B}$ have a beneficial effect on rhabdomyolysis-induced AKI (RI-AKI) by controlling the production of pro-inflammatory cytokines and by regulating macrophage infiltration (44). Shu et al (45) found that LPS can 


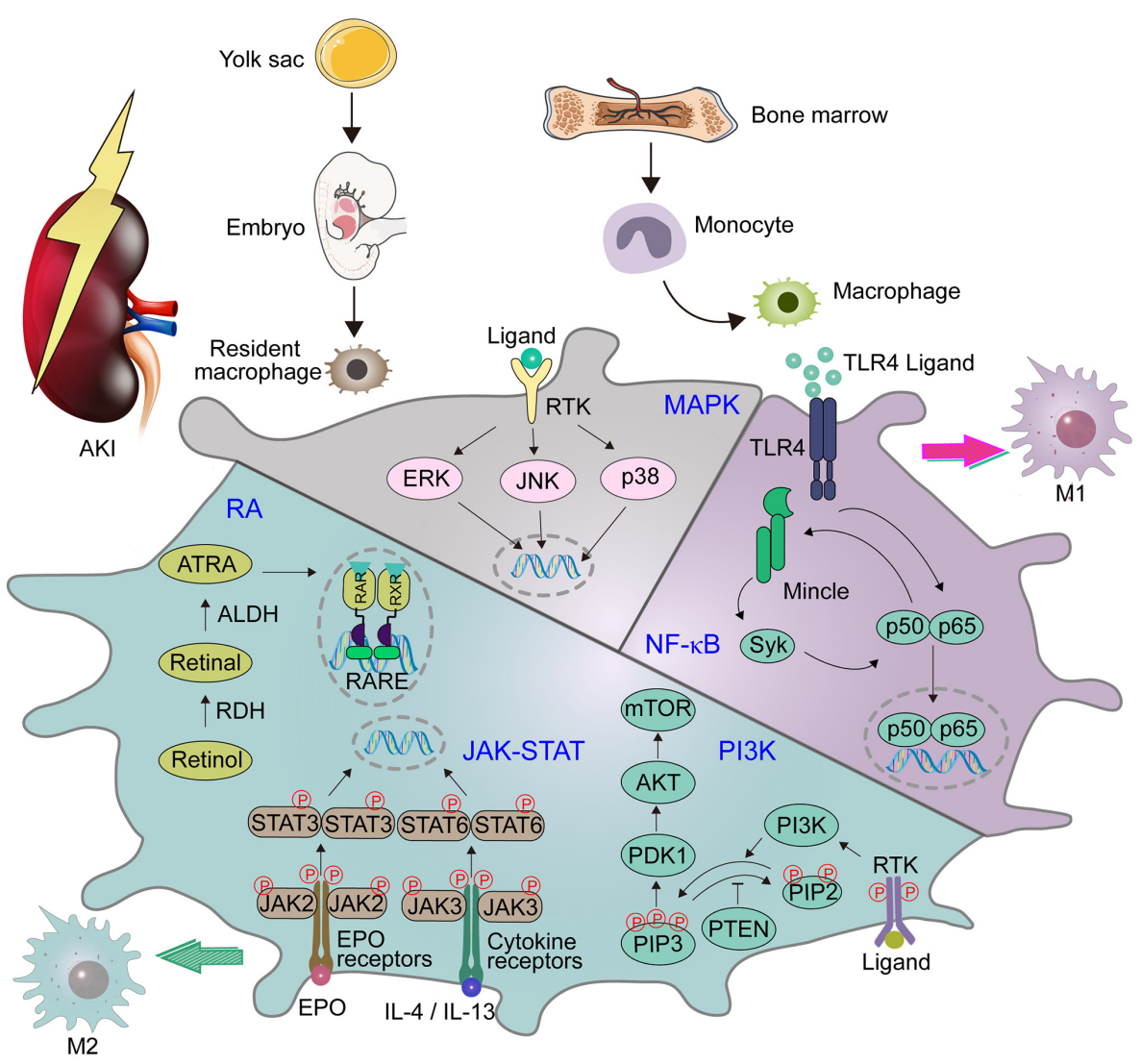

Figure 1. Major signaling pathways of macrophages in AKI. Kidney macrophages are derived from erythro-myeloid progenitors produced in the yolk sac. They are produced in the fetal liver of the embryo, and then settle during organ formation. In the occurrence of inflammation, peripheral macrophages are recruited from the bone marrow to become circulating monocytes. Macrophages have roles in various signaling pathways that are involved in the occurrence

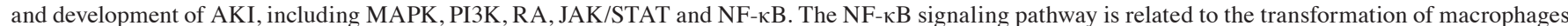
to the M1 phenotype. PI3K, RA and JAK/STAT signaling pathways are related to the transformation of macrophages to the M2 phenotype. AKI, acute kidney injury; MAPK, mitogen-activated protein kinase; PI3K, phosphatidylinositol-3 kinase; RA, retinoic acid; JAK, janus kinase; STAT, signal transducer and activator of transcription; NF- $\mathrm{B}$, nuclear factor- $\mathrm{kB}$; RAR, RA receptor; RARE, RA response elements; EPO, erythropoietin; IL-, interleukin; p-, phosphorylated; ERK, extracellular signal-regulated kinase; TLR4, toll-like receptor 4; PDK1, phosphoinositide-dependent kinase-1; PIP3, phosphatidylinositol (3,4,5)-trisphosphate; PIP2, phosphatidylinositol 4,5-bisphosphate; Mincle, macrophage-inducible C-type lectin; ATRA, all-trans-retinoic acid; ALDH, aldehyde dehydrogenase; RDH, retinol dehydrogenase; RXR, retinoid X receptor; RTK receptor tyrosine kinase.

upregulate the expression of CD38 in macrophages cultured in vitro in a time- and dose-dependent manner. Knocking down or blocking the expression of CD38 in macrophages inhibits the transduction of NF- $\mathrm{BB}$ signals, thereby reducing LPS-induced M1 polarization (45). It has been shown that quercetin may inhibit M1 polarization and NF- $\mathrm{B}$ signal activation in the mouse kidney and spleen macrophages after LPS injection in a mouse model of AKI caused by LPS (45). Previous studies have reported that quercetin inhibits the expression of inflammatory components in macrophages both in vitro and in vivo, thus reducing renal injury $(46,47)$. The mechanism is primarily associated with inhibition of the activity of macrophage-inducible C-type lectin (Mincle) and its downstream signal molecules Syk and NF- $\mathrm{KB}$, whereby it regulates the polarization of macrophages by inhibiting pro-inflammatory M1 macrophages and activating anti-inflammatory M2 macrophages (48). Tan et al (49) have shown that curcumin treatment inhibits M1 macrophages in a Mincle-dependent manner, promoting the phenotypic transformation of macrophages from the M1 phenotype to the M2 phenotype and alleviating M1 macrophage-mediated kidney inflammation through a Syk/NF- $\mathrm{kB}$-dependent mechanism. Hui et al (50) showed that Astragalus propinquus Schischkin and Panax notoginseng could control the activation of Mincle/Syk/NF- $\kappa$ B in vivo and in vitro. The mechanism may involve the inhibition of Mincle, which can decrease the activation of M1 macrophages and increase the activation of M2 macrophages. Zhou et al (51) demonstrated that recombinant human EPO exerts a regulatory effect on macrophages and a beneficial effect on the kidney by lowering the production of pro-inflammatory components via the TLR4/NF- $\mathrm{KB}$ signaling pathway in crush syndrome-induced AKI (Fig. 1). Therefore, EPO has a therapeutic potential for improving the prognosis of patients with AKI.

Janus kinase (JAK)/signal transducer and activator of transcription (STAT) signaling pathway. JAK is a non-receptor tyrosine kinase (52), while STAT molecules are potential nuclear transcription factors that are activated after recruitment of activated receptor complexes (53). The JAK/STAT signaling pathway transduces the downstream signals of a variety of cytokines, which is important in the pathogenesis of immune-related diseases (54). Previous studies have shown that EPO can assist M2 polarization through the JAK2/STAT3/STAT6 signaling pathway, suggesting the immunomodulatory ability of EPO in macrophage activation 
(Fig. 1) $(23,55)$. In addition, the cytokines interleukin (IL)-4 and IL-13 can activate the JAK3/STAT6 signaling pathway (Fig. 1). Deletion of IL-4 and IL-13 will lead to the decrease of the M2a phenotypic markers of tissue repair, which indicates that IL-4 and IL-13 play an essential role in the phenotypic polarization of $\mathrm{M} 2 \mathrm{a}$ macrophages and are important in I/R-AKI recovery (56). Gpnmb is a transmembrane protein that negatively regulates macrophage inflammation (57). It has been observed that the IL-4/STAT6 signaling pathway participates in the process of M2 polarization by Gpnmb (24). This suggests that Gpnmb can be used as a biomarker to protect against AKI by regulating the polarization of macrophages (24).

Mitogen-activated protein kinase (MAPK) signaling pathway. MAPKs are serine-threonine protein kinases. In mammals, MAPKs include c-Jun N-terminal kinase, p38 MAPK and extracellular signal-regulated kinase. Each type exists in different subtypes of the enzyme and can regulate a variety of cellular activities, including proliferation, differentiation and apoptosis (Fig. 1) (58). MAPK participates in the process of sepsis-induced AKI (59,60). A previous study demonstrated that the expression of kidney injury molecule-1 (KIM-1) is significantly increased in both AKI and CKD (61). It has been reported that the MAPK signaling pathway may play a pivotal role in KIM-1-mediated macrophage phenotypic transition and migration (62). Li et al (63) demonstrated that AQP1 exerts a protective effect in the regulation of AKI and attenuates macrophage-mediated inflammation by downregulating the activity of p38 MAPK induced by LPS in RAW264.7 cells. Consequently, pharmacological inhibitors targeting the AQP1-mediated p38 MAPK signaling pathway may be potential treatments for AKI.

$R A$ signaling pathway. RA signaling plays an important role in numerous biological processes (64). RA is synthesized at a specific location, and regulates transcription by interacting with the nuclear RA receptor. It then binds to the RA response elements near the target gene (Fig. 1) (65). It has been found that the RA signaling pathway participates in AKI in mouse and zebrafish models of AKI, limiting the extent of damage and promoting regular repair (66). RA signaling coordinates the dynamic balance of pro-inflammatory M1 and anti-inflammatory M2 macrophages (67). Locally synthesized RA inhibits the pro-inflammatory M1 phenotype, thus reducing macrophage-dependent damage and activating RA signaling in the damaged tubular epithelium, which in turn promotes the selective activation of M2 macrophages (68). It was previously demonstrated that the effect of 4-(phenylthio) butanoic acid (PTBA) on the proliferation of RTECs is dependent on RA signaling (69). The therapeutic characteristics of PTBA are not limited to the kidney, since PTBA also increases the proliferation of cardiomyocytes and reduces fibrosis after heart injury in adult zebrafish. This provides a critical insight into the mechanism by which PTBA enhances repair in AKI (69). In addition, it has also been reported that RA has a beneficial effect on CI-AKI in vivo and in vitro. Specifically, it activates autophagy, inhibits apoptosis and reduces renal fibrosis after CI-AKI. This provides a theoretical basis for RA as a potential drug for the prophylaxis and treatment of CI-AKI (25).

\section{Macrophage-derived mediators in AKI}

Macrophage migration inhibitory factor (MIF). MIF is a crucial effector of the innate immune and inflammatory response $(70,71)$. In contrast to the majority of cytokines, MIF is expressed by the epithelial lining of tissues in direct contact with the external environment as well as by immune and endocrine cells, which can improve the pro-inflammatory function of immune cells (72). MIF promoter polymorphism can predict the occurrence of postoperative AKI (73). A previous study demonstrated that MIF is increased in the urine of patients with acute pyelonephritis (APN), and that the concentration of MIF is associated with the severity of renal injury (74). Thus, MIF is considered to be a potential biomarker in patients with AKI caused by APN. AKI is the most common complication after cardiac surgery (75). It has been reported that MIF is a pressure-regulated cytokine that can protect the heart from myocardial I/R injury (76). MIF can limit the extent of necrotizing nephropathy and reduce oxidative stress effectively by regaining intracellular glutathione and reducing lipid peroxidation (77). MIF has clinical importance as treatment after cardiac surgery, as it may exert a potential nephroprotective effect in AKI (77). Thioredoxin-1 (TRX-1) is a MIF-regulatory protein with redox activity, and has a short residence time in blood (78). Long-acting TRX genetically fused with human serum albumin has the potential to treat RI-AKI by regulating oxidative stress and MIF (79). Previous studies have found that MIF plays a pathogenic role in cisplatin-induced AKI, and MIF can mediate AKI via a CD74/NF- $\kappa$ B-dependent mechanism (Figs. 2 and 3) $(80,81)$. The MIF inhibitor ribosomal protein S19, which targets MIF, may be a potential treatment strategy for AKI (82). Li et al (81) showed that high levels of MIF may be a pathogenic factor for I/R-AKI, and the level of MIF in plasma and urine may be associated with the progression and remission of AKI. Thus, targeting MIF may be a potential treatment for AKI (Table I).

Macrophage CD163. CD163 is a $130 \mathrm{kDa}$ transmembrane receptor protein, which is mainly expressed by M2c macrophages (83). Urinary soluble CD163 (sCD163) may be used as a biomarker in certain renal inflammatory diseases (Fig. 2) (84). Sun et al (85) quantified sCD163 in the urine, and measured macrophage subtypes in the urine and renal biopsies (Table I). The authors found that urinary M1 is associated with interstitial M1 infiltration, while urinary sCD163 level and M2 subtype are positively correlated with infiltrating M2 in the glomeruli. The study by Sun et al (85) also revealed that urinary sCD163 has an improved diagnostic ability in distinguishing the disease etiology than that of traditional AKI urinary [Lipocalin-2 (Lcn-2)/neutropil gelatinase-associated lipocalin (NGAL) and KIM-1], myeloid cell (CD11b) and pan-macrophage (CD68) markers. Rubio-Navarro et al (86) developed a targeted probe for CD163 by magnetic resonance imaging in vivo, thus confirming the presence of CD163-positive macrophages in human RI-AKI. Myoglobin activates early inflammatory M1 reaction and partial transformation to the $\mathrm{M} 2$ phenotype at a later stage, in which the high expression of CD163 is due to activation of heme oxygenase 1 and release of IL-10 (86). Furthermore, the aforementioned study developed gold-coated iron oxide nanoparticles with anti-CD163 antibody as a carrier, 


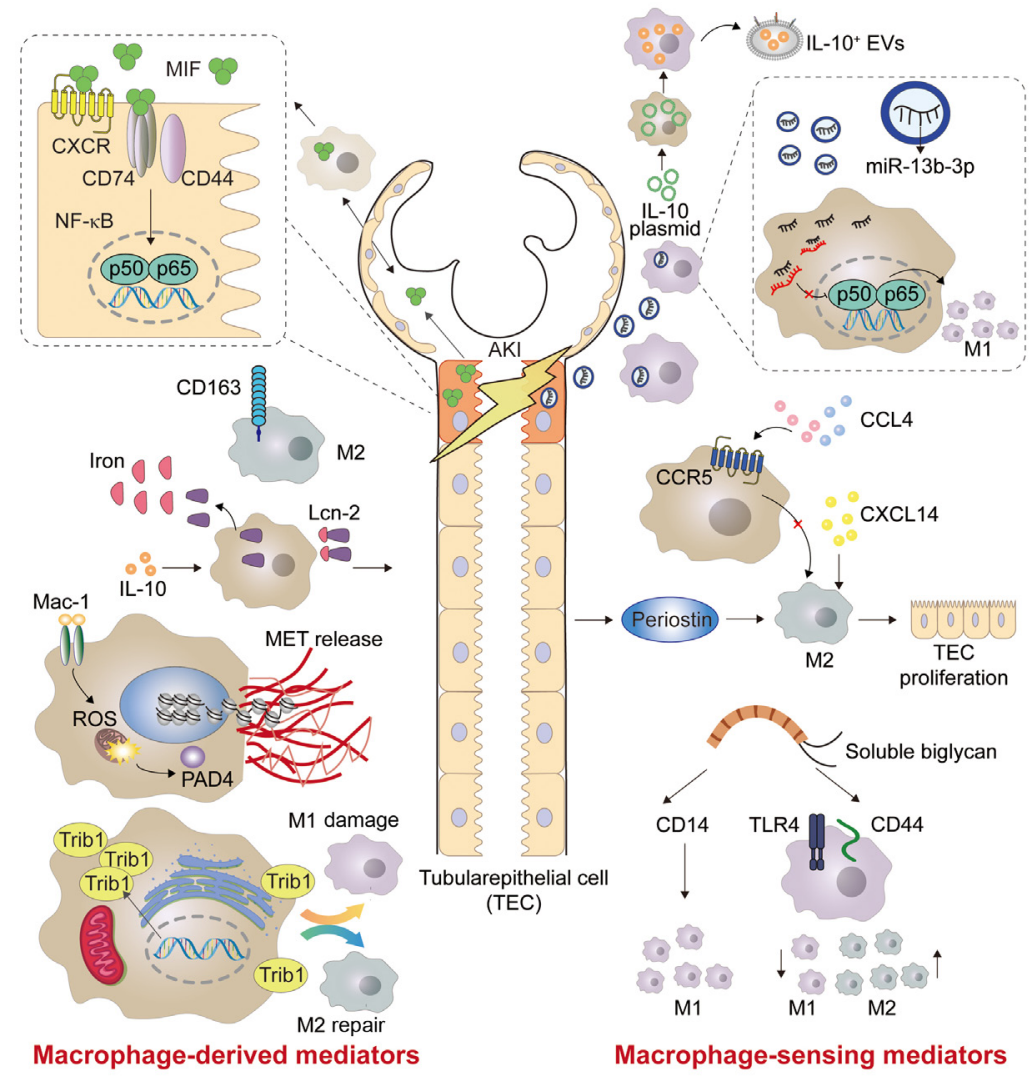

Figure 2. Macrophage-derived mediators and macrophage-sensing mediators in AKI. MIF is expressed in the epithelial lining of tissues, as well as in immune and endocrine cells, and is involved in the mechanisms of NF- $\mathrm{KB}$ signaling. CD163 is a surface marker for M2 macrophages. METs are activated by heme and platelets to release DNA fibers and granular proteins. Trib1 and biglycan are able to adjust the proportion of M1 and M2 macrophages. Exosomal miR-19b-3p from tubular epithelial cells promote the activation of M1 macrophages during AKI. Both CXCL14 and periostin alleviate AKI by increasing the number of

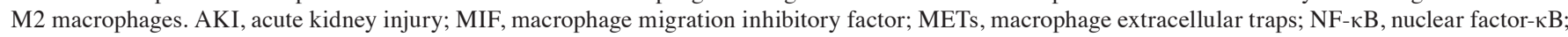
Trib1, tribbles homolog 1; EVs, extracellular vesicles; miR, microRNA; CXCL14, C-X-C motif chemokine ligand 14; CXCR, CXC chemokine receptors; Lcn-2, lipocalin-2; IL-, interleukin; ROS, reactive oxygen species; TEC, tubular epithelial cell; CCL4, C-C motif chemokine 4; TLR4, toll-like receptor 4; CCR5, chemokine receptor 5; PAD4, peptidyl-arginine deiminase 4.

which specifically targeted CD163 in the kidneys of mice injected with glycerol (86). Using probes targeting CD163 macrophages by magnetic resonance imaging could provide valuable information on the cellular composition of kidney lesions in rhabdomyolysis (86).

Lcn-2. Lcn-2/ NGAL, is a member of the lipocalin family, which shares a tight tertiary structure, and ligand binding and post-translational modifications in Lcn-2 leads to diverse Lcn-2 functions (87). Lcn-2 is considered a therapeutic target for AKI, CKD and numerous types of cancer such as breast, esophagus and liver cancer (87-89). Lcn-2 plays a pivotal role in various kidney diseases, such as sepsis and IR (90). After renal injury, Lcn-2 increases rapidly in macrophages, and has highly stable iron binding and transport capacity (Fig. 2). A study by Urbschat et al (91) showed that mouse tubular epithelial cells (TECs) promoted the proliferation of epithelial cells by taking macrophage-derived iron-loaded Lcn-2. Mertens et al (92) concluded that the cellular source of Lcn-2 (TECs or macrophages) and its iron loading determine the biological function of Len-2 in cecal ligation and puncture (CLP)-induced kidney injury. At $24 \mathrm{~h}$ after CLP-induced kidney injury, elevated levels of iron-free Lcn-2 in TECs are primarily considered as markers of kidney injury, whereas elevated levels of iron-loaded Lcn-2 from macrophage sources are associated with markers of recovery (92) (Fig. 2; Table I). In summary, Lcn-2 is involved in renal injury and renal function recovery.

Macrophage extracellular traps (METs). ETs were first identified and described at neutrophils in 2004 (93). Neutrophil ETs (NETs) are generated by neutrophils after releasing granulin and chromatin, which together form extracellular fibers that bind gram-positive and negative bacteria. Similar to NETs, it has been found that a variety of pathogens and chemical stimulants can induce macrophages to produce METs, including gram-positive and -negative bacteria, acid-resistant bacteria, parasites, fungi, and chemical inducers and inhibitors (94). NETs and METs are the main drivers of the self-amplification circuit of necrotizing inflammation (Fig. 2) (95). A recent study reported the mechanism of muscle damage leading to renal dysfunction, and identified the formation of METs as a novel type of pathogenic driver and a potential therapeutic target (96). A previous study also found that macrophages participate in the pathological process of AKI caused by rhabdomyolysis (23). Macrophages release METs containing DNA fibers and granular proteins in a mouse model of rhabdomyolysis. During rhabdomyolysis, platelets activated by heme released by necrotizing muscle cells increased MET production by increasing intracellular 


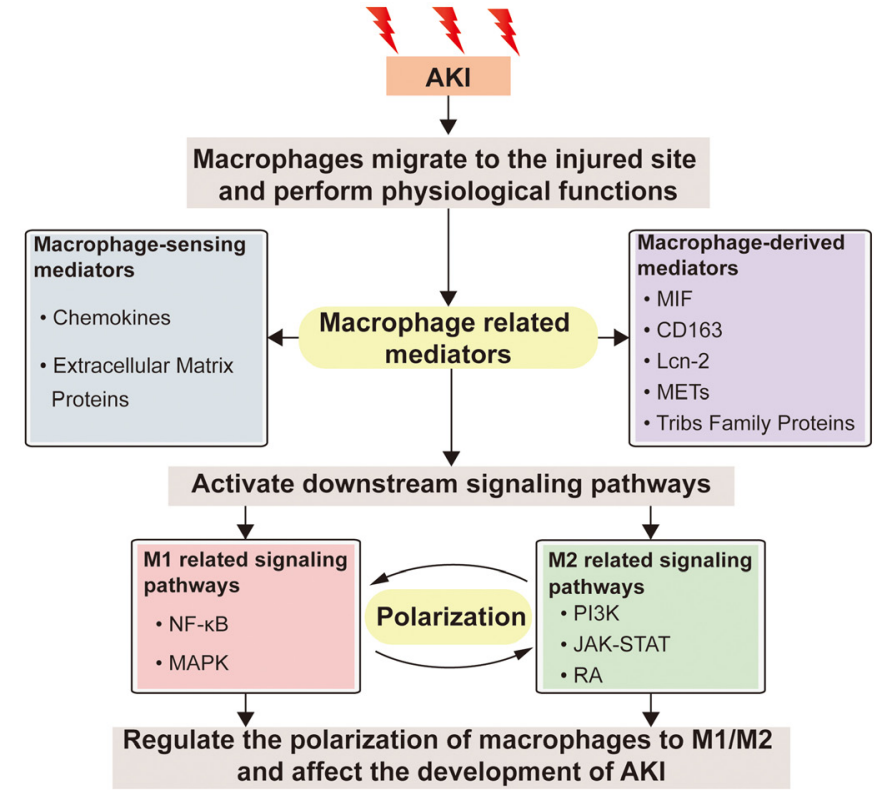

Figure 3. Relationship between macrophage-related mediators and downstream signaling pathways after AKI. When AKI occurs, macrophages migrate to the injured site and activate downstream signal pathways through the production of macrophage-related mediators to regulate the phenotype transformation of macrophages and exert their physiological functions Macrophage-related mediators include macrophage-derived mediators and macrophage-sensing mediators, which activate signaling pathways related to M1/M2 macrophages, regulate the polarization of macrophages, and affect the development of AKI. AKI, acute kidney injury; MIF, macrophage migration inhibitory factor; Lcn-2, lipocalin-2; METs, macrophage extracellular traps; TRIBS, tribbles homolog; MAPK, mitogen-activated protein kinase; PI3K, phosphatidylinositol-3 kinase; RA, retinoic acid; JAK, janus kinase STAT, signal transducer and activator of transcription; NF- $\kappa \mathrm{B}$, nuclear factor- $\mathrm{\kappa} \mathrm{B}$.

reactive oxygen species production and histone citrullination. It was the first study to demonstrate that METs and platelets act as sensors for myoglobin-derived heme in RI-AKI (97) (Fig. 3; Table I). The study reported that the mechanism of METs participating in AKI is useful for the pathological study of the disease, and may provide novel ideas for the treatment of AKI (98).

Tribbles homolog (TRIB) family proteins. TRIB family members are pseudokinase proteins that are conserved among species and are associated with various human diseases such as leukemia and metabolic disorders (99). Members of the TRIB family are basic regulators of the cell cycle, proliferation, differentiation, metabolism and cellular stress (100). Among them, TRIB1 can control the differentiation of M2 macrophages (101), and its deficiency results in a marked reduction of M2 macrophages not only in the bone marrow, but also in adipose, lung and spleen tissue (102). TRIB1 may widely control the polarization of M1/M2 macrophages via the JAK/STAT signaling pathway (Figs. 2 and 3) (103). It was previously demonstrated that, during the period of moderate AKI adaptive recovery induced by I/R, TRIB1 regulates the proliferation of renal tubular cells by affecting the polarization of macrophages, thus playing a role in renal recovery and regeneration (104) (Table I). Therefore, TRIB1 may be a promising target for improving adaptive renal repair after I/R injury.

\section{Macrophage-sensing mediators in AKI}

Chemokines. Chemokines are a large family of small, secreted proteins that play a fundamental role in the development and dynamic balance of the immune system. Chemokine signals are sent through cell surface G-protein coupled heptahelical chemokine receptors (105). Chemokines play a long-term role in physiological and pathological regulation by inducing macrophage differentiation and polarization (106). C-X-C motif chemokine ligand 14 (CXCL14) is a relatively new CXC type of chemokine, which is constitutively expressed in breast, kidney and other epithelial tissues (107). A previous study indicated that its overexpression inhibits M1 polarization and increases M2 polarization. This suggested that the overexpression of CXCL14 may reduce AKI caused by sepsis by downregulating the production of macrophage-derived cytokines (Fig. 2) (108). Chemokine receptor 5 (CCR5) is a pivotal regulator of the inflammatory cascade response of macrophages in the kidney, and its deficiency contributes to the activation of M2 macrophages (Fig. 2; Table I) (109). Therefore, blocking CCR5 may be helpful in the treatment of I/R-AKI. It has been observed that the deficiency of C-C motif CCR 2 can prevent the migration of $\mathrm{Ly} 6 \mathrm{C}^{+}$macrophages from the bone marrow to the injured site, thus reducing ischemic-AKI in mice (110). In the activation of the renin-angiotensin system, $\mathrm{C}-\mathrm{C}$ motif chemokine ligand 5 (CCL5) paradoxically limits the accumulation of macrophages in the injured kidney by inhibiting the pro-inflammatory effect of CCL2 (111).

Extracellular matrix proteins. Periostin is a type of cellular-matrix protein of $90 \mathrm{kDa}$ in size, which is highly expressed in bone and tooth tissues (112). As a recently identified biomarker of renal disease, periostin is mainly associated with CKD (113). However, Kormann et al (114) demonstrated that periostin can increase the production of M2 macrophages in the stage of AKI repair, as well as assist the repair of RTECs by promoting the proliferation of macrophages and the secretion of regenerating factors in the kidney (Table I).

Biglycan is a small proteoglycan, abundant in leucine, which acts as a danger signal derived from the extracellular matrix in a soluble form, and it is a high-affinity ligand of CD14 in macrophages (115). The lack of CD14 prevents biglycan-mediated cytokine expression, macrophage recruitment and polarization to M1 macrophages. It also leads to a decrease in the activation of the biglycan-TLR2/4 signaling pathway, thus improving renal function (Figs. 2 and 3) (116). Poluzzi et al (117) showed that CD44 is a novel biglycan signal coreceptor, and its interaction with biglycan is necessary for TLR4/CD44-dependent autophagy in macrophages. Interfering with the interaction between biglycan and specific TLR coreceptors may constitute a novel therapeutic intervention to reduce renal inflammation and injury. Specifically, the role of biglycan in inflammation exceeds its function as a typical danger signal, and it is the first ligand, among numerous types of DAMPs, that has been reported to promote the polarization of M1 and M2 macrophages simultaneously through the transduction of different coreceptor signals (118) (Table I). Biglycan binds to CD14 to promote tissue inflammation, while it binds to CD44 to induce autophagy in M1 macrophages and then promote tissue remodeling (119). 


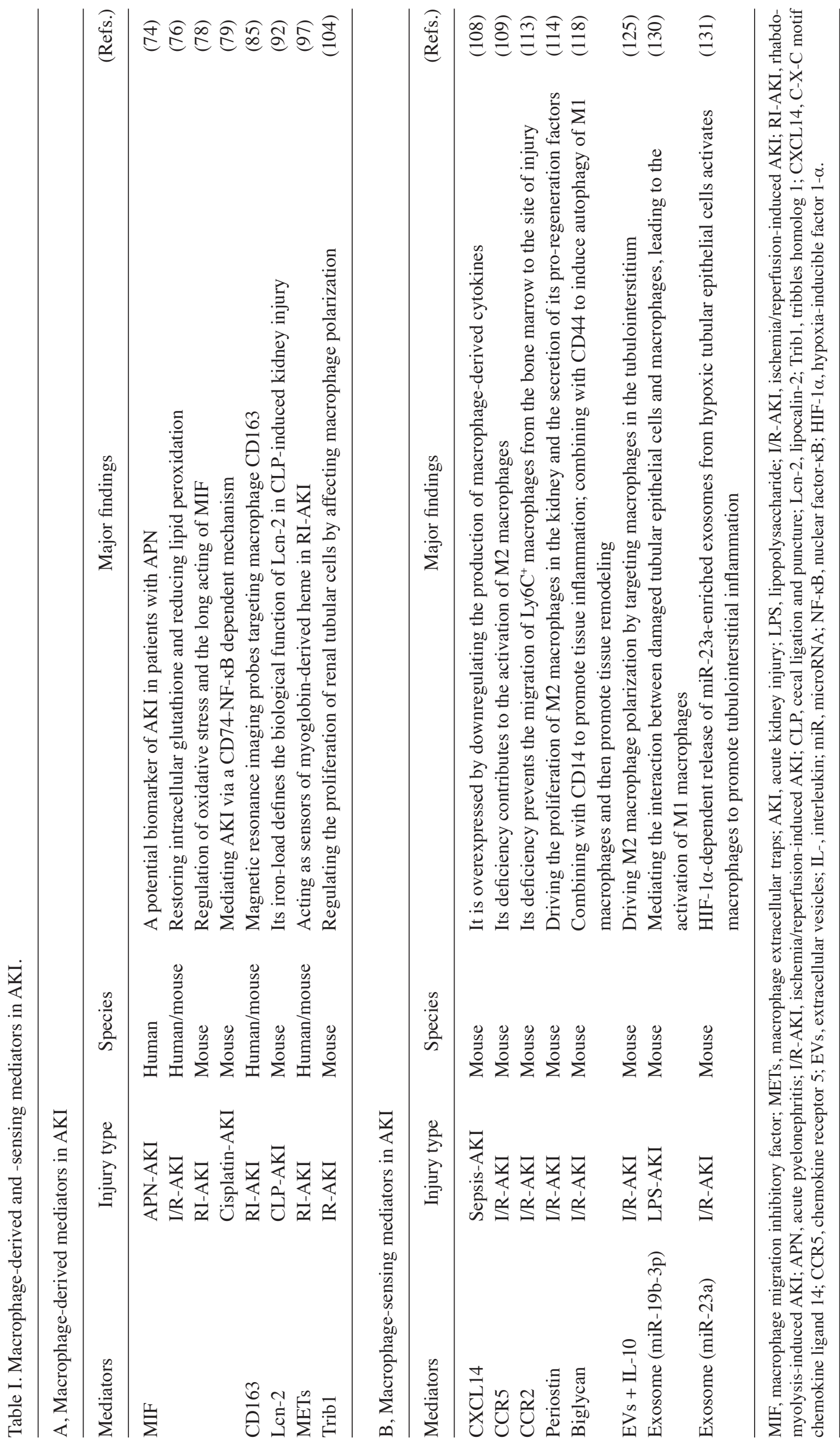


Extracellular vesicles (EVs) refer to the membrane structures released by all types of cells through different biogenic pathways (120). EVs are intercellular messengers that are involved in a wide range of physiological and pathological processes (121). EVs have the advantages of exhibiting stable physical and chemical properties (122). Therefore, EVs have been used as natural carriers in drug delivery systems in recent years (123). It was previously shown that macrophage-derived EVs have a pro-inflammatory effect (124). By contrast, Tang et al (125) demonstrated that macrophages secrete IL-10 ${ }^{+}$ EVs under dexamethasone stimulation, which can promote the transformation of macrophages into the M2 phenotype (Fig. 2; Table I).

Exosomes are nanoscale EVs that play a key role in intercellular communication and signal transduction. They also play a pivotal role in the processes of inflammation and immune response (126). In recent years, a growing body of evidence has found that exosomes participate in the pathogenesis of renal disease $(127,128)$. Among them, exosomal microRNA (miR)-19b-3p may be a promising therapeutic target for renal disease (129). Tubulointerstitial inflammation is an important pathological feature of AKI. Lv et al (130) found that exosomal miR-19b-3p mediates the interaction between injured RTECs and macrophages, which leads to the activation of M1 macrophages and indicates that the exosome/miR-19b-3p/suppressor of the cytokine signaling axis plays a pivotal role in the development of renal tubulointerstitial inflammation (Fig. 2; Table I). Furthermore, previous studies have demonstrated that the release of hypoxia-inducible factor- $1 \alpha$-dependent miR-23a-enriched exosomes from hypoxic TECs can activate macrophages and promote tubulointerstitial inflammation (131) (Table I). In addition, blocking the exosome-mediated transfer of miR-23a between RTECs and macrophages may be an innovative method for enhancing renal tubulointerstitial inflammation. In summary, exosomes are involved in adjusting the routine function of the kidney, as well as in promoting and inhibiting various pathophysiological reactions (121).

\section{Conclusions}

AKI may be caused by a variety of factors and injuries, with a high incidence among critically ill patients (132). When AKI occurs, macrophages, as one of the most significant types of immune cells, are recruited to the injury site to perform their physiological functions. M1 macrophages can aggravate the inflammatory response in order to eliminate potential pathogens. However, excessive inflammatory response aggravates cell and tissue damage. M2 macrophages promote immune suppression and tissue regeneration $(28,133)$. Therefore, the application of M2 macrophages and macrophage-modulating agents is a current research hotspot in the treatment of AKI $(11,23)$. It was previously demonstrated that M2 macrophages can cause the transition from AKI to CKD, which increases the risk of CKD among AKI survivors (34). Recent studies on the role of macrophages in the pathogenesis of AKI suggest the complication and diversity of macrophage activation and the functional status after renal injury $(134,135)$. The multifaceted role of macrophages in AKI is mainly mediated by PI3K, JAK/STAT, RA, NF- $\kappa$ B, MAPK and other signaling pathways (Fig. 1), which may regulate macrophage polarization or oxidative stress $(42,44,56,59,68)$. Among them, the RA signaling pathway is considered to be the current research hotspot, because it not only has an immunosuppressive effect, but also participates in the inflammatory response. Elucidating the mechanism of the RA signaling pathway in AKI and its association with macrophages has great clinical application prospects (136). Macrophage-derived and -sensing mediators such as MIF, CD163, Lcn-2, METs, TRIB, and biglycan (Fig. 2; Table I) also play an essential role in AKI. Fig. 3 shows the association between macrophage-associated mediators and downstream signaling pathways after AKI. However, the signaling pathway that macrophage mediators participate in may be a complex network, and further research is needed to verify whether there is interaction between different pathways. A thorough understanding of the biological role of macrophages in the process of injury and repair is a necessary condition for understanding the limitations and further applicability of macrophages in therapy. Therefore, future studies should elucidate the mechanism and timing of macrophage polarization, as well as the precise regulatory mechanisms of macrophages in the occurrence and development of AKI, which will contribute to the understanding and identification of novel therapeutic targets for AKI.

\section{Acknowledgements}

Not applicable.

\section{Funding}

This research was supported by grants from The Open Fund of State Key Laboratory of Medicinal Chemical Biology (Nankai University; grant no. 2020010) and The Tianjin University 'Double first class' construction talent start-up fund.

\section{Availability of data and materials}

Not applicable.

\section{Authors' contributions}

NL and JC conceived and designed the review, researched the literature and wrote the manuscript. PW contributed to literature collection and figure preparation. $\mathrm{SH}, \mathrm{HF}$ and $\mathrm{YG}$ designed the study, critically revised and supervised the manuscript. Data authentication is not applicable. All authors read and approved the final manuscript.

\section{Ethics approval and consent to participate}

Not applicable.

\section{Patient consent for publication}

Not applicable.

\section{Competing interests}

The authors declare that they have no competing interests. 


\section{References}

1. Messerer DAC, Halbgebauer R, Nilsson B, Pavenstädt H, Radermacher P and Huber-Lang M: Immunopathophysiology of trauma-related acute kidney injury. Nat Rev Nephrol 17: 91-111, 2021.

2. Eppensteiner J, Davis RP, Barbas AS, Kwun J and Lee J: Immunothrombotic activity of damage-associated molecular patterns and extracellular vesicles in secondary organ failure induced by trauma and sterile insults. Front Immunol 9: 190, 2018.

3. Wynn TA, Chawla A and Pollard JW: Macrophage biology in development, homeostasis and disease. Nature 496: 445-455, 2013.

4. Ginhoux F and Guilliams M: Tissue-resident macrophage ontogeny and homeostasis. Immunity 44: 439-449, 2016.

5. Gomez Perdiguero E, Klapproth K, Schulz C, Busch K, Azzoni E, Crozet L, Garner H, Trouillet C, de Bruijn MF, Geissmann F and Rodewald HR: Tissue-resident macrophages originate from yolk-sac-derived erythro-myeloid progenitors. Nature 518 547-551, 2015

6. Okabe Y and Medzhitov R: Tissue biology perspective on macrophages. Nat Immunol 17: 9-17, 2016.

7. Kim SY and Nair MG: Macrophages in wound healing: Activation and plasticity. Immunol Cell Biol 97: 258-267, 2019.

8. Wynn TA and Vannella KM: Macrophages in tissue repair, regeneration, and fibrosis. Immunity 44: 450-462, 2016

9. Vannella KM and Wynn TA: Mechanisms of organ injury and repair by macrophages. Annu Rev Physiol 79: 593-617, 2017.

10. Stein M, Keshav S, Harris N and Gordon S: Interleukin 4 potently enhances murine macrophage mannose receptor activity: A marker of alternative immunologic macrophage activation. J Exp Med 176: 287-292, 1992.

11. Chen T, Cao Q, Wang Y and Harris DCH: M2 macrophages in kidney disease: Biology, therapies, and perspectives. Kidney Int 95: 760-773, 2019.

12. Verdeguer F and Aouadi M: Macrophage heterogeneity and energy metabolism. Exp Cell Res 360: 35-40, 2017.

13. Smigiel KS and Parks WC: Macrophages, wound healing, and fibrosis: Recent insights. Curr Rheumatol Rep 20: 17, 2018.

14. Shapouri-Moghaddam A,Mohammadian S, Vazini H,Taghadosi M Esmaeili SA, Mardani F, Seifi B, Mohammadi A, Afshari JT and Sahebkar A: Macrophage plasticity, polarization, and function in health and disease. J Cell Physiol 233: 6425-6440, 2018.

15. Funes SC, Rios M, Escobar-Vera J and Kalergis AM: Implications of macrophage polarization in autoimmunity. Immunology 154 $186-195,2018$

16. Levey AS and James MT: Acute kidney injury. Ann Intern Med 167: ITC66-ITC80, 2017.

17. Gameiro J, Fonseca JA, Outerelo C and Lopes JA: Acute kidney injury: From diagnosis to prevention and treatment strategies. J Clin Med 9: 1704, 2020.

18. Stewart IJ, Sosnov JA, Howard JT and Chung KK: Acute kidney injury in critically injured combat veterans: A retrospective cohort study. Am J Kidney Dis 68: 564-570, 2016.

19. Rahbar Saadat Y, Hosseiniyan Khatibi SM, Ardalan M, Barzegari A and Zununi Vahed S: Molecular pathophysiology of acute kidney injury: The role of sirtuins and their interactions with other macromolecular players. J Cell Physiol 236: 3257-3274, 2021

20. Maekawa H, Inoue T, Ouchi H, Jao TM, Inoue R, Nishi H, Fujii R, Ishidate F, Tanaka T, Tanaka Y, et al: Mitochondrial damage causes inflammation via cGAS-STING signaling in acute kidney injury. Cell Rep 29: 1261-1273.e6, 2019.

21. Ward DB and Valentovic MA: Contrast induced acute kidney injury and direct cytotoxicity of iodinated radiocontrast media on renal proximal tubule cells. J Pharmacol Exp Ther 370: 160-171, 2019.

22. Bellomo R, Kellum JA and Ronco C: Acute kidney injury. Lancet 380: 756-766, 2012.

23. Wang S, Zhang C, Li J, Niyazi S, Zheng L, Xu M, Rong R, Yang $\mathrm{C}$ and Zhu T: Erythropoietin protects against rhabdomyolysis-induced acute kidney injury by modulating macrophage polarization. Cell Death Dis 8: e2725, 2017.

24. Zhou L, Zhuo H, Ouyang H, Liu Y, Yuan F, Sun L, Liu F and Liu H: Glycoprotein non-metastatic melanoma protein b (Gpnmb) is highly expressed in macrophages of acute injured kidney and promotes M2 macrophages polarization. Cell Immunol 316: 53-60, 2017.
25. Wu J, Wan X, Zhang H, Li W, Ma M, Pan B, Liang X and Cao C: Retinoic acid attenuates contrast-induced acute kidney injury in a miniature pig model. Biochem Biophys Res Commun 512: 163-169, 2019.

26. Huen SC and Cantley LG: Macrophage-mediated injury and repair after ischemic kidney injury. Pediatr Nephrol 30: 199-209, 2015.

27. Jang HR and Rabb H: Immune cells in experimental acute kidney injury. Nat Rev Nephrol 11: 88-101, 2015.

28. Huen SC and Cantley LG: Macrophages in renal injury and repair. Annu Rev Physiol 79: 449-469, 2017.

29. Han HI, Skvarca LB, Espiritu EB, Davidson AJ and Hukriede NA: The role of macrophages during acute kidney injury: Destruction and repair. Pediatr Nephrol 34: 561-569, 2019

30. Singbartl K, Formeck CL and Kellum JA: Kidney-immune system crosstalk in AKI. Semin Nephrol 39: 96-106, 2019.

31. Li X, Mu G, Song C, Zhou L, He L, Jin Q and Lu Z: Role of M2 Macrophages in Sepsis-induced acute kidney injury. Shock 50: 233-239, 2018.

32. Mao R, Wang C, Zhang F, Zhao M, Liu S, Liao G, Li L, Chen Y, Cheng J, Liu J and Lu Y: Peritoneal M2 macrophage transplantation as a potential cell therapy for enhancing renal repair in acute kidney injury. J Cell Mol Med 24: 3314-3327, 2020.

33. Kim MG, Lim K, Lee YJ, Yang J, Oh SW, Cho WY and Jo SK: M2 macrophages predict worse long-term outcomes in human acute tubular necrosis. Sci Rep 10: 2122, 2020.

34. Baek JH: The impact of versatile macrophage functions on acute kidney injury and its outcomes. Front Physiol 10: 1016, 2019.

35. Wu H, Wang Y, Zhang Y, Xu F, Chen J, Duan L, Zhang T, Wang J and Zhang F: Breaking the vicious loop between inflammation, oxidative stress and coagulation, a novel anti-thrombus insight of nattokinase by inhibiting LPS-induced inflammation and oxidative stress. Redox Biol 32: 101500, 2020.

36. Yao W, Guo A, Han X, Wu S, Chen C, Luo C, Li H, Li S and Hei Z: Aerosol inhalation of a hydrogen-rich solution restored septic renal function. Aging (Albany NY) 11: 12097-12113, 2019.

37. Kaneda MM, Messer KS, Ralainirina N, Li H, Leem CJ, Gorjestani S, Woo G, Nguyen AV, Figueiredo CC, Foubert P, et al: $\mathrm{PI} 3 \mathrm{~K} \gamma$ is a molecular switch that controls immune suppression. Nature 539: 437-442, 2016.

38. Qin S, Li J, Zhou C, Privratsky B, Schettler J, Deng X, Xia Z, Zeng Y, Wu $\mathrm{H}$ and Wu M: SHIP-1 regulates phagocytosis and M2 polarization through the PI3K/Akt-STAT5-Trib1 circuit in pseudomonas aeruginosa infection. Front Immunol 11: 307, 2020.

39. Duan Y, Zheng H, Li Z, Yao Y, Ding J, Wang X, Nakkala JR, Zhang D, Wang Z, Zuo X, et al: Unsaturated polyurethane films grafted with enantiomeric polylysine promotes macrophage polarization to a M2 phenotype through PI3K/Akt1/mTOR axis. Biomaterials 246: 120012, 2020.

40. An C, Wen J, Hu Z, Mitch WE and Wang Y: Phosphoinositide 3-kinase $\gamma$ deficiency attenuates kidney injury and fibrosis in angiotensin II-induced hypertension. Nephrol Dial Transplant 35: $1491-1500,2020$

41. Amano MT, Castoldi A, Andrade-Oliveira V, Latancia MT, Terra FF, Correa-Costa M, Breda CNS, Felizardo RJF, Pereira WO, da Silva MB, et al: The lack of PI3K $\gamma$ favors M1 macrophage polarization and does not prevent kidney diseases progression. Int Immunopharmacol 64: 151-161, 2018.

42. Liu C, Li B, Tang K, Dong X, Xue L, Su G and Jin Y: Aquaporin 1 alleviates acute kidney injury via PI3K-mediated macrophage M2 polarization. Inflamm Res 69: 509-521, 2020.

43. Markó L, Vigolo E, Hinze C, Park JK, Roël G, Balogh A, Choi M, Wübken A, Cording J, Blasig IE, et al: Tubular epithelial NF-kappaB activity regulates ischemic AKI. J Am Soc Nephrol 27: 2658-2669, 2016.

44. Huang RS, Zhou JJ, Feng YY, Shi M, Guo F, Gou SJ, Salerno S, Ma L and Fu P: Pharmacological inhibition of macrophage toll-like receptor 4/Nuclear Factor-kappa B alleviates rhabdomyolysis-induced acute kidney injury. Chin Med J (Engl) 130 2163-2169, 2017.

45. Shu B, Feng Y, Gui Y, Lu Q, Wei W, Xue X, Sun X, He W, Yang J and Dai C: Blockade of CD38 diminishes lipopolysaccharide-induced macrophage classical activation and acute kidney injury involving NF-кB signaling suppression. Cell Signal 42: 249-258, 2018.

46. Wang Y, Quan F, Cao Q, Lin Y, Yue C, Bi R, Cui X, Yang H, Yang Y, Birnbaumer L, et al: Quercetin alleviates acute kidney injury by inhibiting ferroptosis. J Adv Res 28: 231-243, 2020. 
47. Lu H, Wu L, Liu L, Ruan Q, Zhang X, Hong W, Wu S, Jin G and Bai Y: Quercetin ameliorates kidney injury and fibrosis by modulating M1/M2 macrophage polarization. Biochem Pharmacol 154: 203-212, 2018

48. Tan RZ, Wang C, Deng C, Zhong X, Yan Y, Luo Y, Lan HY, He T and Wang L: Quercetin protects against cisplatin-induced acute kidney injury by inhibiting Mincle/Syk/NF- $\mathrm{B}$ signaling maintained macrophage inflammation. Phytother Res 34: 139-152, 2020.

49. Tan RZ, Liu J, Zhang YY, Wang HL, Li JC, Liu YH, Zhong X, Zhang YW, Yan Y, Lan HY and Wang L: Curcumin relieved cisplatin-induced kidney inflammation through inhibiting Mincle-maintained M1 macrophage phenotype. Phytomedicine 52: 284-294, 2019.

50. Hui D, Rui-Zhi T, Jian-Chun L, Xia Z, Dan W, Jun-Ming F and Li W: Astragalus propinquus Schischkin and Panax notoginseng (A\&P) compound relieved cisplatin-induced acute kidney injury through inhibiting the mincle maintained macrophage inflammation. J Ethnopharmacol 252: 112637, 2020.

51. Zhou J, Bai Y, Jiang Y, Tarun P, Feng Y, Huang R and Fu P Immunomodulatory role of recombinant human erythropoietin in acute kidney injury induced by crush syndrome via inhibition of the TLR4/NF- $\kappa \mathrm{B}$ signaling pathway in macrophages. Immunopharmacol Immunotoxicol 42: 37-47, 2020.

52. Xu P, Shen P, Yu B, Xu X, Ge R, Cheng X, Chen Q, Bian J, Li Z and Wang J: Janus kinases (JAKs): The efficient therapeutic targets for autoimmune diseases and myeloproliferative disorders. Eur J Med Chem 192: 112155, 2020.

53. Bousoik E and Montazeri Aliabadi H: 'Do We Know Jack' About JAK? A closer Look at JAK/STAT signaling pathway. Front Oncol 8: 287, 2018.

54. Banerjee S, Biehl A, Gadina M, Hasni S and Schwartz DM: JAK-STAT signaling as a target for inflammatory and autoimmune diseases: Current and future prospects. Drugs 77: 521-546, 2017.

55. Zhu M, Wang L, Yang J, Xie K, Zhu M, Liu S, Xu C, Wang J, Gu L, Ni Z, et al: Erythropoietin ameliorates lung injury by accelerating pulmonary endothelium cell proliferation via janus kinase-signal transducer and activator of transcription 3 pathway after kidney ischemia and reperfusion injury. Transplant Proc 51: 972-978, 2019

56. Zhang MZ, Wang X, Wang Y, Niu A, Wang S, Zou C and Harris RC: IL-4/IL-13-mediated polarization of renal macrophages/dendritic cells to an M2a phenotype is essential for recovery from acute kidney injury. Kidney Int 91: 375-386, 2017

57. van der Lienden MJC, Gaspar P, Boot R, Aerts JMFG and van Eijk M: Glycoprotein non-metastatic protein B: An emerging biomarker for lysosomal dysfunction in macrophages. Int $\mathrm{J}$ Mol Sci 20: 66, 2018

58. Kim EK and Choi EJ: Compromised MAPK signaling in human diseases: An update. Arch Toxicol 89: 867-882, 2015

59. Ren Q, Guo F, Tao S, Huang R, Ma L and Fu P: Flavonoid fisetin alleviates kidney inflammation and apoptosis via inhibiting Src-mediated NF- $\mathrm{BB}$ p 65 and MAPK signaling pathways in septic AKI mice. Biomed Pharmacother 122: 109772, 2020.

60. Sun S, Wang J, Wang J, Wang F, Yao S and Xia H: Maresin 1 mitigates sepsis-associated acute kidney injury in mice via inhibition

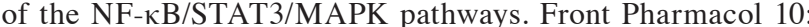
$1323,2019$.

61. Wen Y and Parikh CR: Current concepts and advances in biomarkers of acute kidney injury. Crit Rev Clin Lab Sci 1-24, 2021 (Epub ahead of print).

62. Tian L, Shao X, Xie Y, Wang Q, Che X, Zhang M, Xu W, $\mathrm{Xu} \mathrm{Y}$, Mou S and Ni Z: Kidney injury molecule-1 is elevated in nephropathy and mediates macrophage activation via the mapk signalling pathway. Cell Physiol Biochem 41: 769-783, 2017.

63. Li B, Liu C, Tang K, Dong X, Xue L, Su G, Zhang W and Jin Y: Aquaporin-1 attenuates macrophage-mediated inflammatory responses by inhibiting p38 mitogen-activated protein kinase activation in lipopolysaccharide-induced acute kidney injury. Inflamm Res 68: 1035-1047, 2019.

64. Conserva MR, Anelli L, Zagaria A, Specchia G and Albano F: The pleiotropic role of retinoic acid/retinoic acid receptors signaling: From vitamin A metabolism to gene rearrangements in acute promyelocytic leukemia. Int J Mol Sci 20: 2921, 2019.

65. Cunningham TJ and Duester G: Mechanisms of retinoic acid signalling and its roles in organ and limb development. Nat Rev Mol Cell Biol 16: 110-123, 2015.

66. Ghyselinck NB and Duester G: Retinoic acid signaling pathways Development 146: dev167502, 2019.
67. Vellozo NS, Pereira-Marques ST, Cabral-Piccin MP, Filardy AA, Ribeiro-Gomes FL, Rigoni TS, DosReis GA and Lopes MF: All-Trans Retinoic Acid Promotes an M1- to M2-Phenotype shift and inhibits macrophage-mediated immunity to leishmania major. Front Immunol 8: 1560, 2017

68. Chiba T, Skrypnyk NI, Skvarca LB, Penchev R, Zhang KX, Rochon ER, Fall JL, Paueksakon P, Yang H, Alford CE, et al: Retinoic acid signaling coordinates macrophage-dependent injury and repair after AKI. J Am Soc Nephrol 27: 495-508, 2016.

69. Brilli Skvarca L, Han HI, Espiritu EB, Missinato MA, Rochon ER, McDaniels MD, Bais AS, Roman BL, Waxman JS, Watkins SC, et al: Enhancing regeneration after acute kidney injury by promoting cellular dedifferentiation in zebrafish. Dis Model Mech 12: dmm037390, 2019.

70. Harris J, VanPatten S, Deen NS, Al-Abed Y and Morand EF: Rediscovering MIF: New tricks for an old cytokine. Trends Immunol 40: 447-462, 2019.

71. Kang I and Bucala R: The immunobiology of MIF: Function, genetics and prospects for precision medicine. Nat Rev Rheumatol 15: 427-437, 2019.

72. Calandra $\mathrm{T}$ and Roger T: Macrophage migration inhibitory factor: A regulator of innate immunity. Nat Rev Immunol 3: 791-800, 2003

73. Averdunk L, Bernhagen J, Fehnle K, Surowy H, Lüdecke HJ, Mucha S, Meybohm P, Wieczorek D, Leng L, Marx G, et al: The macrophage migration inhibitory factor (MIF) promoter polymorphisms (rs3063368, rs755622) predict acute kidney injury and death after cardiac surgery. J Clin Med 9: 2936, 2020.

74. Hong MY, Tseng CC, Chuang CC, Chen CL, Lin SH and Lin CF: Urinary macrophage migration inhibitory factor serves as a potential biomarker for acute kidney injury in patients with acute pyelonephritis. Mediators Inflamm 2012: 381358, 2012.

75. Wu B, Chen J and Yang Y: Biomarkers of acute kidney injury after cardiac surgery: A narrative review. Biomed Res Int 2019: $7298635,2019$.

76. Fuhrman DY and Kellum JA: Epidemiology and pathophysiology of cardiac surgery-associated acute kidney injury. Curr Opin Anaesthesiol 30: 60-65, 2017.

77. Stoppe C, Averdunk L, Goetzenich A, Soppert J, Marlier A Kraemer S, Vieten J, Coburn M, Kowark A, Kim BS, et al: The protective role of macrophage migration inhibitory factor in acute kidney injury after cardiac surgery. Sci Transl Med 10: eaan4886, 2018.

78. Leaver SK, MacCallum NS, Pingle V, Hacking MB, Quinlan GJ, Evans TW and Burke-Gaffney A: Increased plasma thioredoxin levels in patients with sepsis: Positive association with macrophage migration inhibitory factor. Intensive Care Med 36 336-341, 2010.

79. Nishida K, Watanabe H, Ogaki S, Kodama A, Tanaka R, Imafuku T, Ishima Y, Chuang VT, Toyoda M, Kondoh M, et al: Renoprotective effect of long acting thioredoxin by modulating oxidative stress and macrophage migration inhibitory factor against rhabdomyolysis-associated acute kidney injury. Sci Rep 5: 14471, 2015.

80. Li J, Tang Y, Tang PMK, Lv J, Huang XR, Carlsson-Skwirut C, Da Costa L, Aspesi A, Fröhlich S, Szczęśniak P, et al: Blocking macrophage migration inhibitory factor protects against cisplatin-induced acute kidney injury in mice. Mol Ther 26: 2523-2532, 2018

81. Li JH, Tang Y, Lv J, Wang XH, Yang H, Tang PMK, Huang XR, He ZJ, Zhou ZJ, Huang QY, et al: Macrophage migration inhibitory factor promotes renal injury induced by ischemic reperfusion. J Cell Mol Med 23: 3867-3877, 2019.

82. Lv J, Huang XR, Klug J, Fröhlich S, Lacher P, Xu A, Meinhardt A and Lan HY: Ribosomal protein S19 is a novel therapeutic agent in inflammatory kidney disease. Clin Sci (Lond) 124: 627-637, 2013.

83. Matsushita T and Takehara K: Soluble CD163 is a potential biomarker in systemic sclerosis. Expert Rev Mol Diagn 19: 197-199, 2019.

84. Mejia-Vilet JM, Zhang XL, Cruz C, Cano-Verduzco ML, Shapiro JP, Nagaraja HN, Morales-Buenrostro LE and Rovin BH: Urinary soluble CD163: A novel noninvasive biomarker of activity for lupus nephritis. J Am Soc Nephrol 31: 1335-1347, 2020.

85. Sun PP, Zhou XJ, Su JQ, Wang C, Yu XJ, Su T, Liu G, Wang SX, Nie J and Yang L: Urine macrophages reflect kidney macrophage content during acute tubular interstitial and glomerular injury. Clin Immunol 205: 65-74, 2019. 
86. Rubio-Navarro A, Carril M, Padro D, Guerrero-Hue M, Tarín C, Samaniego R, Cannata P, Cano A, Villalobos JM, Sevillano ÁM, et al: CD163-Macrophages are involved in rhabdomyolysis-induced kidney injury and may be detected by MRI with targeted gold-coated iron oxide nanoparticles. Theranostics 6: 896-914, 2016.

87. Li D, Yan Sun W, Fu B, Xu A and Wang Y: Lipocalin-2-The myth of its expression and function. Basic Clin Pharmacol Toxicol 127: 142-151, 2020.

88. Rahimi S, Roushandeh AM, Ahmadzadeh E, Jahanian-Najafabadi A and Roudkenar MH: Implication and role of neutrophil gelatinase-associated lipocalin in cancer: Lipocalin-2 as a potential novel emerging comprehensive therapeutic target for a variety of cancer types. Mol Biol Rep 47: 2327-2346, 2020.

89. Santiago-Sánchez GS, Pita-Grisanti V, Quiñones-Díaz B, Gumpper K, Cruz-Monserrate Z and Vivas-Mejía PE: Biological functions and therapeutic potential of lipocalin 2 in cancer. Int J Mol Sci 21: 4365, 2020.

90. Desanti De Oliveira B, Xu K, Shen TH, Callahan M, Kiryluk K, D'Agati VD, Tatonetti NP, Barasch J and Devarajan P: Molecular nephrology: Types of acute tubular injury. Nat Rev Nephrol 15: 599-612, 2019.

91. Urbschat A, Thiemens AK, Mertens C, Rehwald C, Meier JK, Baer PC and Jung M: Macrophage-secreted Lipocalin-2 promotes regeneration of injured primary murine renal tubular epithelial cells. Int J Mol Sci 21: 2038, 2020.

92. Mertens C, Kuchler L, Sola A, Guiteras R, Grein S, Brüne B, von Knethen A and Jung M: Macrophage-derived iron-bound lipocalin-2 correlates with renal recovery markers following sepsis-induced kidney damage. Int J Mol Sci 21: 7527, 2020.

93. Brinkmann V, Reichard U, Goosmann C, Fauler B, Uhlemann Y, Weiss DS, Weinrauch Y and Zychlinsky A: Neutrophil extracellular traps kill bacteria. Science 303: 1532-1535, 2004.

94. Doster RS, Rogers LM, Gaddy JA and Aronoff DM: Macrophage extracellular traps: A scoping review. J Innate Immun 10: 3-13, 2018

95. Nakazawa D, Marschner JA, Platen L and Anders HJ: Extracellular traps in kidney disease. Kidney Int 94: 1087-1098, 2018.

96. Hartl D: Macrophages and platelets join forces to release kidney-damaging DNA traps. Nat Med 24: 128-129, 2018.

97. Okubo K, Kurosawa M, Kamiya M, Urano Y, Suzuki A Yamamoto K, Hase K, Homma K, Sasaki J, Miyauchi H, et al: Macrophage extracellular trap formation promoted by platelet activation is a key mediator of rhabdomyolysis-induced acute kidney injury. Nat Med 24: 232-238, 2018.

98. Lichtman A: The kidney gets caught in a macrophage trap. Sci Immunol 3: eaat 3745, 2018.

99. Satoh T, Kidoya H, Naito H, Yamamoto M, Takemura N, Nakagawa K, Yoshioka Y, Morii E, Takakura N, Takeuchi O and Akira S: Critical role of Trib1 in differentiation of tissue-resident M2-like macrophages. Nature 495: 524-528, 2013.

100. Eyers PA, Keeshan K and Kannan N: Tribbles in the 21 st century: The evolving roles of tribbles pseudokinases in biology and disease. Trends Cell Biol 27: 284-298, 2017.

101. Liu ZZ, Han ZD, Liang YK, Chen JX, Wan S, Zhuo YJ, Cai ZD, Deng YL, Lin ZY, Mo RJ, et al: TRIB1 induces macrophages to M2 phenotype by inhibiting IKB-zeta in prostate cancer. Cell Signal 59: 152-162, 2019

102. Shiraishi M, Shintani Y, Shintani Y, Ishida H, Saba R, Yamaguchi A, Adachi H, Yashiro K and Suzuki K: Alternatively activated macrophages determine repair of the infarcted adult murine heart. J Clin Invest 126: 2151-2166, 2016.

103. Arndt L, Dokas J, Gericke M, Kutzner CE, Müller S, Jeromin F, Thiery $\mathrm{J}$ and Burkhardt R: Tribbles homolog 1 deficiency modulates function and polarization of murine bone marrow-derived macrophages. J Biol Chem 293: 11527-11536, 2018

104. Xie X, Yang X, Wu J, Ma J, Wei W, Fei X and Wang M: Trib1 contributes to recovery from ischemia/reperfusion-induced acute kidney injury by regulating the polarization of renal macrophages. Front Immunol 11: 473, 2020.

105. Hughes CE and Nibbs RJB: A guide to chemokines and their receptors. FEBS J 285: 2944-2971, 2018.

106. Ruytinx P, Proost P, Van Damme J and Struyf S: Chemokine-induced macrophage polarization in inflammatory conditions. Front Immunol 9: 1930, 2018.

107. Lu J, Chatterjee M, Schmid H, Beck S and Gawaz M: CXCL14 as an emerging immune and inflammatory modulator. J Inflamm (Lond) 13: 1, 2016.
108. Lv J, Wu ZL, Gan Z, Gui P and Yao SL: CXCL14 overexpression attenuates sepsis-associated acute kidney injury by inhibiting proinflammatory cytokine production. Mediators Inflamm 2020: 2431705, 2020

109. Yoo KD, Cha RH, Lee S, Kim JE, Kim KH, Lee JS, Kim DK, Kim YS and Yang SH: Chemokine receptor 5 blockade modulates macrophage trafficking in renal ischaemic-reperfusion injury. J Cell Mol Med 24: 5515-5527, 2020

110. Yang Q, Wang Y, Pei G, Deng X, Jiang H, Wu J, Zhou C, Guo Y, Yao Y, Zeng R and Xu G: Bone marrow-derived Ly6C(-) macrophages promote ischemia-induced chronic kidney disease. Cell Death Dis 10: 291, 2019.

111. Rudemiller NP, Patel MB, Zhang JD, Jeffs AD, Karlovich NS, Griffiths R, Kan MJ, Buckley AF, Gunn MD and Crowley SD: C-C Motif Chemokine 5 Attenuates Angiotensin II-Dependent kidney injury by limiting renal macrophage infiltration. Am J Pathol 186: 2846-2856, 2016.

112. Prakoura N and Chatziantoniou C: Periostin in kidney diseases. Cell Mol Life Sci 74: 4315-4320, 2017.

113. Wallace DP: Periostin in the kidney. Adv Exp Med Biol 1132: 99-112, 2019.

114. Kormann R, Kavvadas P, Placier S, Vandermeersch S, Dorison A, Dussaule JC, Chadjichristos CE, Prakoura N and Chatziantoniou C: Periostin promotes cell proliferation and macrophage polarization to drive repair after AKI. J Am Soc Nephrol 31: 85-100, 2020.

115. Roedig H, Nastase MV, Wygrecka M and Schaefer L: Breaking down chronic inflammatory diseases: The role of biglycan in promoting a switch between inflammation and autophagy. FEBS J 286: 2965-2979, 2019.

116. Roedig H, Nastase MV, Frey H, Moreth K, Zeng-Brouwers J, Poluzzi C, Hsieh LT, Brandts C, Fulda S, Wygrecka M and Schaefer L: Biglycan is a new high-affinity ligand for CD14 in macrophages. Matrix Biol 77: 4-22, 2019.

117. Poluzzi C, Nastase MV, Zeng-Brouwers J, Roedig H, Hsieh LT, Michaelis JB, Buhl EM, Rezende F, Manavski Y, Bleich A, et al: Biglycan evokes autophagy in macrophages via a novel CD44/Toll-like receptor 4 signaling axis in ischemia/reperfusion injury. Kidney Int 95: 540-562, 2019.

118. Meissner M, Viehmann SF and Kurts C: DAMPening sterile inflammation of the kidney. Kidney Int 95: 489-491, 2019.

119. Roedig H, Damiescu R, Zeng-Brouwers J, Kutija I, Trebicka J, Wygrecka M and Schaefer L: Danger matrix molecules orchestrate CD14/CD44 signaling in cancer development. Semin Cancer Biol 62: 31-47, 2020

120. Zhang PL and Liu ML: Extracellular vesicles mediate cellular interactions in renal diseases-Novel views of intercellular communications in the kidney. J Cell Physiol 2021 (Epub ahead of print)

121. Rigalli JP, Barros ER, Sommers V, Bindels RJM and Hoenderop JGJ: Novel aspects of extracellular vesicles in the regulation of renal physiological and pathophysiological processes. Front Cell Dev Biol 8: 244, 2020.

122. Wu P, Zhang B, Ocansey DKW, Xu W and Qian H: Extracellular vesicles: A bright star of nanomedicine. Biomaterials 269 120467, 2020.

123. Zhang X, Zhang H, Gu J, Zhang J, Shi H, Qian H, Wang D, $\mathrm{Xu}$ W, Pan J and Santos HA: Engineered extracellular vesicles for cancer therapy. Adv Mater e2005709, 2021 (Epub ahead of print).

124. Quaglia M, Dellepiane S, Guglielmetti G, Merlotti G, Castellano G and Cantaluppi V: Extracellular vesicles as mediators of cellular crosstalk between immune system and kidney graft. Front Immunol 11: 74, 2020.

125. Tang TT, Wang B, Wu M, Li ZL, Feng Y, Cao JY, Yin D, Liu H, Tang RN, Crowley SD, et al: Extracellular vesicle-encapsulated IL-10 as novel nanotherapeutics against ischemic AKI. Sci Adv 6: eaaz0748, 2020

126. Console L, Scalise M and Indiveri C: Exosomes in inflammation and role as biomarkers. Clin Chim Acta 488: 165-171, 2019.

127. Ståhl AL, Johansson K, Mossberg M, Kahn R and Karpman D: Exosomes and microvesicles in normal physiology, pathophysiology, and renal diseases. Pediatr Nephrol 34: 11-30, 2019.

128. Thongboonkerd V: Roles for exosome in various kidney diseases and disorders. Front Pharmacol 10: 1655, 2020.

129. Wang ZW and Zhu X: Exosomal miR-19b-3p communicates tubular epithelial cells and M1 macrophage. Cell Death Dis 10: 762,2019 
130. Lv LL, Feng Y, Wu M, Wang B, Li ZL, Zhong X, Wu WJ, Chen J, Ni HF, Tang TT, et al: Exosomal miRNA-19b-3p of tubular epithelial cells promotes M1 macrophage activation in kidney injury. Cell Death Differ 27: 210-226, 2020.

131. Li ZL, Lv LL, Tang TT, Wang B, Feng Y, Zhou LT, Cao JY, Tang $\mathrm{RN}, \mathrm{Wu} \mathrm{M}$, Liu $\mathrm{H}$, et al: HIF-1 $\alpha$ inducing exosomal microRNA-23a expression mediates the cross-talk between tubular epithelial cells and macrophages in tubulointerstitial inflammation. Kidney Int 95: 388-404, 2019.

132. Harrois A, Soyer B, Gauss T, Hamada S, Raux M and Duranteau J: Prevalence and risk factors for acute kidney injury among trauma patients: A multicenter cohort study. Crit Care 22: 344, 2018

133. Zuk A and Bonventre JV: Acute kidney injury. Annu Rev Med 67: 293-307, 2016.
134. Wen Y and Crowley SD: The varying roles of macrophages in kidney injury and repair. Curr Opin Nephrol Hypertens 29: 286-292, 2020.

135. Tang PM, Nikolic-Paterson DJ and Lan HY: Macrophages: Versatile players in renal inflammation and fibrosis. Nat Rev Nephrol 15: 144-158, 2019.

136. Poeck H, Bscheider M, Gross O, Finger K, Roth S, Rebsamen M, Hannesschläger N, Schlee M, Rothenfusser S, Barchet W, et al: Recognition of RNA virus by RIG-I results in activation of CARD9 and inflammasome signaling for interleukin 1 beta production. Nat Immunol 11: 63-69, 2010.

cc) (i) $\Theta$ This work is licensed under a Creative Commons Attribution-NonCommercial-NoDerivatives 4.0 International (CC BY-NC-ND 4.0) License. 